\title{
Local Delivery of Minocycline and Vorinostat Targets the Tumor Microenvironment to Inhibit the Recurrence of Glioma
}

\author{
Gang Zhao $\mathbb{D}^{1, *}$ \\ Jun Jia ${ }^{1, *}$ \\ Lansheng Wang ${ }^{1, *}$ \\ Yongkang Zhang' \\ Han Yang' \\ Yang Lu' \\ Rutong $\mathrm{Yu}^{\mathrm{l}, 2}$ \\ Hongmei Liu (D) ${ }^{1-3}$ \\ Yufu Zhu ${ }^{1,2}$ \\ 'Institute of Nervous System Diseases, \\ Xuzhou Medical University, Xuzhou \\ 221002, People's Republic of China; \\ ${ }^{2}$ Department of Neurosurgery, The \\ Affiliated Hospital of Xuzhou Medical \\ University, Xuzhou 221002, People's \\ Republic of China; ${ }^{3}$ Department of \\ Neurosurgery, The Third People's \\ Hospital Affiliated of Xuzhou Medical \\ University, Xuzhou, People's Republic of \\ China
}

*These authors contributed equally to this work
This article was published in the following Dove Press journal: OncoTargets and Therapy

Background: Postoperative recurrence is the main reason for poor clinical outcomes in glioma patients, so preventing tumor recurrence is crucial in the management of gliomas.

Methods: In this study, the expression of matrix metalloproteinases (MMPs) in normal tissues was detected via RNA-seq analysis. Glioma cases from the public databases (The Cancer Genome Atlas (TCGA), the Chinese Glioma Genome Atlas (CGGA)) were included in this study. The hydrogel contains minocycline (Mino) and vorinostat (Vor) (G/Mino+Vor) was formed under $365 \mathrm{~nm}$ when the photoinitiator was added. High-performance liquid chromatography (HPLC) was used to assess the release of drugs in the G/Mino+Vor hydrogel. An MTT assay was used to explore the biosecurity of GelMA. Immunohistochemistry, ELISA, and TUNEL assays were used to demonstrate the antitumor effect of the G/Mino +Vor hydrogel.

Results: We successfully developed a G/Mino+Vor hydrogel. The experiments in vitro and in vivo confirmed the MMPs-responsive delivery of minocycline and vorinostat in hydrogel and the anti-glioma effect on an incomplete tumor operation model, which indicated that the G/Mino+Vor hydrogel effectively inhibited the recurrence of glioma after surgery.

Conclusion: In summary, the G/Mino+Vor hydrogel could continuously release drugs and improve the therapy effects against recurrent glioma.

Keywords: minocycline, vorinostat, recurrent glioma, hydrogel, tumor microenvironmennt

\section{Introduction}

Malignant gliomas are recognized as the most aggressive tumors in central nervous system (CNS). ${ }^{1,2}$ Despite standard therapy including surgery, chemoand radiotherapy have been widely adopted, the average survival time is only about 15 months, whereas the 5-year survival rate is only $4-5 \%,{ }^{3-5}$ Irrespective of the current aggressive and multi-model first-line approaches of diagnosis and treatment, recurrence of glioblastoma (GBM) is practically inevitable, with an average time of recurrence is less than 10 months. $^{2,6-8}$ Various treatments modalities are utilized, including repeat resection, irradiation, and systemic agents; however, the prognosis for these patients remains discouraging. The need for more innovative therapeutic strategies to expedite recurrent glioma therapeutics is imperative.

Clinical studies have found $80-90 \%$ of recurrent glioma occur near the primary site, suggesting that residual tumor cells caused most glioma recurrence, ${ }^{5,9,10}$ To break through the physiological barrier, gliomas secrete extracellular matrix (ECM)
Correspondence: Yufu Zhu; Hongmei Liu Tel +86 I8052268I67 Email fugle99@163.com; liuhongmei816@sina.com 
degrading MMPs, ${ }^{11-13}$ which are also upregulated after tissue injury. Therefore, it may play an important role in the recurrence of gliomas after surgery. One agent showing promise is tetracycline antibiotics targeting MMPs, minocycline. Researchers have found that minocycline reduces MMPs expression from microglia by blocking toll-like receptor 2 (TLR2) signaling and p38 mitogen-activated protein kinase (MAPK) pathway. ${ }^{14,15}$ Unfortunately, the bloodbrain barrier (BBB) restricts the concentrations of minocycline delivered within the tumor microenvironment (TME). ${ }^{16}$ Higher doses of minocycline are not prescribed due to severe liver and kidney toxicity. ${ }^{17}$

The immune response may be suppressed by polarization of macrophages within TME into M2 macrophages, which hamper immune responses by releasing anti-inflammatory cytokines and angiogenic factors. ${ }^{18-21}$ Thus, repolarizing M2 to proinflammatory M1 could improve the immune response in TME. ${ }^{22,23}$ Previous studies have shown the ability of vorinostat to have anti-tumor effects by manipulating macrophages. ${ }^{24-26}$ However, due to poor solubility and stability, vorinostat was found to be difficult to be absorbed into the body, resulting in poor bioavailability in vivo. Moreover, vorinostat has significant adverse effects, including nausea, fatigue, and thrombocytopenia. ${ }^{27,28}$

Because of these problems in the clinical application of minocycline and vorinostat, we developed a drug-loaded hydrogel with gelatin methacryloyl (GelMA). It allows for the delivery of high local drug doses to the residual infiltrative cells with minimized systemic toxicity. Due to the presence of methacrylamide groups, the mixture solution was immediately cross-linked under UV light when photoinitiator was added, and ahydrogel was formed. ${ }^{29-31}$ GelMA can release drugs owing to its biodegradability in $\mathrm{TME}^{32,33}$ (Sch.1A). Then, minocycline would hamper the expression of MMPs from microglia, and vorinostat helps to repolarize $\mathrm{M} 2$ to proinflammatory M1 (Sch.1B). Minocycline can act on TME together with vorinostat to improve the efficiency of anti-tumor therapy.

\section{Materials and Methods}

\section{Materials}

GelMA was purchased from Tissueink (Wenzhou, China). Minocycline and vorinostat were purchased from Dalian Meilun (Dalian, China).

Cell lines (GL261, HA1800) were obtained from the Cell Resource Center of Shanghai Institutes for Biological Sciences, Chinese Academy of Sciences (Shanghai,
China). Male mice (C57BL/6 at 6-8 weeks of age) were purchased from HFK Bioscience (Beijing, China). All animal experiments were conducted in accordance with protocols approved by the Experimental Animal Ethics Committee of Xuzhou Medical University (Ethics Number: 202007A260).

Samples from GBM and grade II-III glioma patients were obtained from the Affiliated Hospital of Xuzhou Medical University (Xuzhou, China). The pathological diagnosis was completed at the Department of Pathology, the Affiliated Hospital of Xuzhou Medical University. This study was approved by the Ethics Committee of Clinical Trials of Affiliated Hospital of Xuzhou Medical University. Informed consent was obtained from all participants (Ethics Number: XYFY2016-KL030-0L).

\section{Bioinformatics Analysis}

The clinical data of GBM patients were obtained from two independent data portals (CGGA and TCGA). A total of 349 samples were obtained from TCGA (https://tcga-data. nci.nih.gov/tcga/), and the survival curves of patients were obtained with Betastasis (http://www.betastasis.com/) for clinical correlation. A total of $325 \mathrm{GBM}$ samples with clinical data were obtained from the CGGA (http://www. cgga.org.cn/) cohort to explore the differences in MMP2 and MMP9 among different histological types and WHO grades of glioma.

The RNA transcriptomic data of 117 samples were obtained from TCGA to perform immune cell infiltration analysis. In this routine, we applied the CIBERSORT algorithm (https://cibersort.stanford.edu/) to estimate the immune infiltration subtypes in the GBM microenvironment.

\section{Synthesis and Drug Release of G/Mino +Vor Hydrogels}

GelMA (50 mg) and UV photoinitiator $(5 \mathrm{mg})$ were mixed, followed by the addition of $1 \mathrm{~mL}$ ultrapure sterile water to dissolve it, then minocycline $(10 \mathrm{mg})$ and vorinostat (4 mg) were added to the mixture solution, and UV irradiation $(365 \mathrm{~nm})$ was performed for $15 \mathrm{~s}$ to prepare the hydrogel.

A $100 \mu \mathrm{L}$ G/Mino+Vor hydrogel was placed in adialysis tube, and then moved into a $15 \mathrm{~mL}$ centrifuge tube containing $10 \mathrm{~mL}$ PBS. Human MMP2 (100ng/mL), MMP9 $(100 \mathrm{ng} / \mathrm{mL})$, or lipase $(5000 \mathrm{U} / \mathrm{mL})$ were added at $37^{\circ} \mathrm{C}$. Fifty microliters of dialyzate was taken at each 
time point, and its content was measured by HPLC, and the release curve was drawn according to the regulations. The column of minocycline is Thermo ODS-C18, the mobile phase is $0.2 \mathrm{moL} / \mathrm{L}$ ammonium oxalate- dimethylformamide-0.01 moL/L disodium EDTA (550:200:250), the detection wavelength is $280 \mathrm{~nm}$, the injection volume is $20 \mu \mathrm{L}$, flow rate is $1.5 \mathrm{~mL} / \mathrm{min}$. The column of vorinostat was Thermo ODS-C18, mobile phase was $20 \mathrm{mmol} / \mathrm{L}$ potassium dihydrogen phosphate solution-acetonitrile (700:300), detection wavelength was $242 \mathrm{~nm}$, injection volume was $20 \mu \mathrm{L}$, and flow rate was $1.5 \mathrm{~mL} / \mathrm{min}$.

Standard curve for minocycline. $\mathrm{Y}=0.1423 \mathrm{x}-0.1431$, $\mathrm{R}^{2}=0.9997$.

Standard curve for vorinostat. $\mathrm{Y}=0.2517 \mathrm{x}+0.0233$, $\mathrm{R}^{2}=0.9995$.

\section{Cytotoxicity Assay}

Detection of cytotoxicity of hydrogel carriers using the MTT method. Briefly, GL261 and HA1800 cells were trypsinized and cultured at a density of $5 \times 10^{3}$ per well and spread on 96 -well plates containing $10 \%$ fetal bovine serum (FBS) high-sugar Dulbecco's Modified Eagle's Medium (DMEM) for $24 \mathrm{~h}$. The medium was replaced with FBS-free high-sugar medium. Soaking solution of $1 \mathrm{~mL}$ GelMA hydrogel in PBS buffer at different time points was added. Simultaneously, in another set of experiments, different concentrations of GelMA were added to the culture medium for assessment. After $24 \mathrm{~h}, 20 \mu \mathrm{L} 0.5$ $\mathrm{mg} / \mathrm{mL}$ MTT solution was added to each well and incubated for $4 \mathrm{~h}$. Thereafter, all solutions were carefully removed and $150 \mu \mathrm{L}$ DMSO was added to each well to dissolve the formazan (precipitate). The optical density (OD) was measured by measuring the absorbance at 570 $\mathrm{nm}$ with a microplate reader.

\section{Anti-Tumor Efficacy in vivo and Toxicity Evaluation}

Briefly, $5 \times 10^{5}$ GL261 glioma cells were resuspended in 5 $\mu \mathrm{L}$ Leibovitz's L-15 medium and injected into the right forebrain of 8-week-old mice. On day 10 after tumor implantation, mice bearing tumors were randomly divided into 5 groups (12 mice per group). Mice were gas anesthetized and fixed in a stereotaxic apparatus. A $1.0 \mathrm{~cm}$ incision was made along the midline of the previous surgical scar, and a $1.0 \mathrm{~cm}$ circular skull window was subsequently drilled with a drill to expose the brain tissue. A biopsy punch with a diameter of $2 \mathrm{~mm}$ was inserted at a $5 \mathrm{~mm}$ depth. The biopsy punch was rotated to cut the glioma tissue for $15 \mathrm{~s}$ and aspirate the tumor and brain tissue using a vacuum pump. After hemostasis was compressed, hydrogels containing different drugs (PBS, GelMA, G/ Mino, G/Vor, G/Mino + Vor) were injected into the tumor cavity after surgery. Later suture the wound. Then, we recorded the weight of mice and the survival rate after administration. On day 28 after glioma transplantation, 3 mice in each group were euthanized. The main organs and brain tissues of the dead mice were collected and fixed with $10 \%$ formalin. The fixed organs and brain tissue were embedded in paraffin and stained with H\&E, observed, and photographed with a microscope.

\section{Immunohistochemistry}

To assess the expression of MMPs in tumor tissues. The glioma tissue sections were dewaxed in xylene and rehydrated with gradient ethanol. Next, the sections were incubated in $4 \% \mathrm{H}_{2} \mathrm{O}_{2}$ for $20 \mathrm{~min}$ and then in $3 \%$ albumin from bovine serum (BSA) for $60 \mathrm{~min}$. The sections were incubated with rabbit primary antibodies against MMP2 (1:100, Abcam, Cambridge, MA, USA) or MMP9 (1:100) at $4{ }^{\circ} \mathrm{C}$ overnight. The sections were then incubated with anti-rabbit secondary antibody (Zhongshan Golden Bridge, Beijing, China) for $30 \mathrm{~min}$. After counterstaining with diaminobenzidine (DAB) and hematoxylin, the sections were dehydrated according to gradient ethanol, and then the coverslips were observed and quantified using a microscope. The images were analyzed using Image Pro Plus 6.0 software.

\section{Enzyme-Linked Immunosorbent Assay}

In order to confirm the repolarizing effect of M2 to M1, the markers of M1 (tumor necrosis factor (TNF) $-\alpha$, interleukin (IL) -6) and the markers of M2 (IL-4 and IL-10) were assessed with enzyme-linked immunosorbent assay (ELISA). Briefly, 3 mice from each group were anesthetized using an overdose of $4 \%$ chloral hydrate and killed on day 21. Brain tissues were collected, homogenized in $\mathrm{PBS}$, and centrifuged at $4^{\circ} \mathrm{C}$ and $8000 \mathrm{rpm}$ for $10 \mathrm{~min}$. The supernatant was collected and diluted with an ELISA diluent buffer. The protein content of each homogenate sample was normalized to total protein levels per sample using a BCA kit. 
A
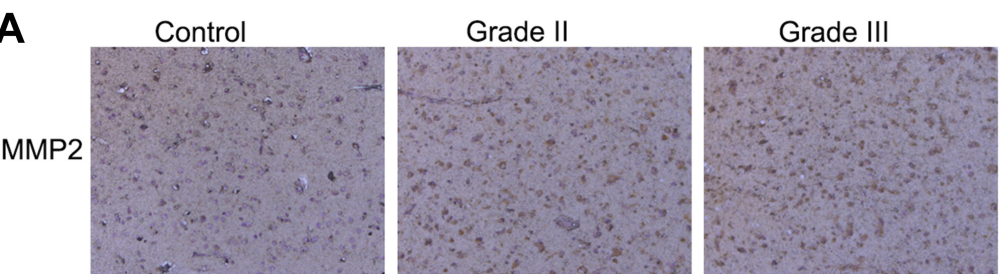

Grade IV
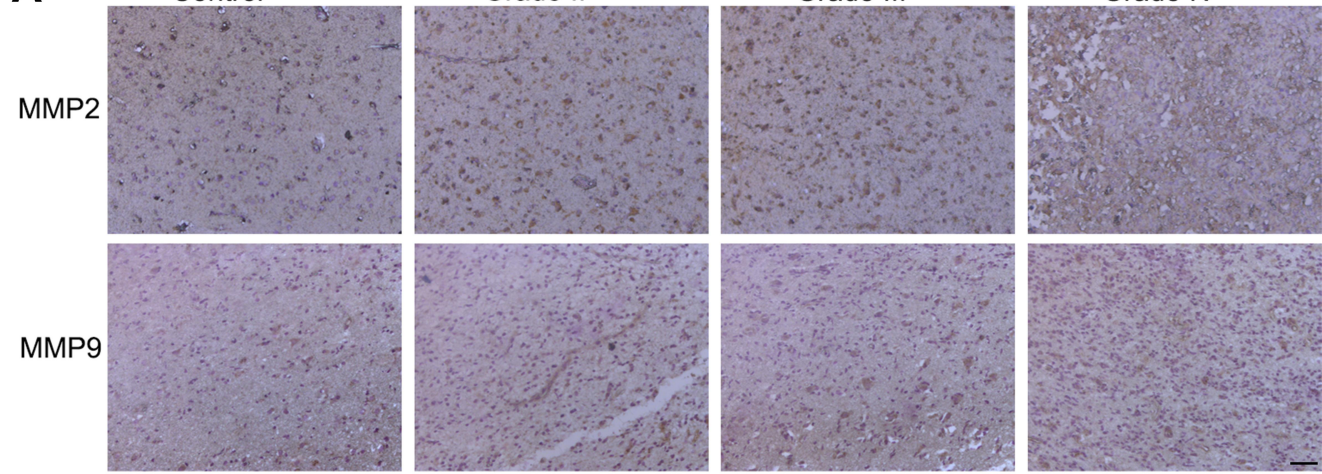

B

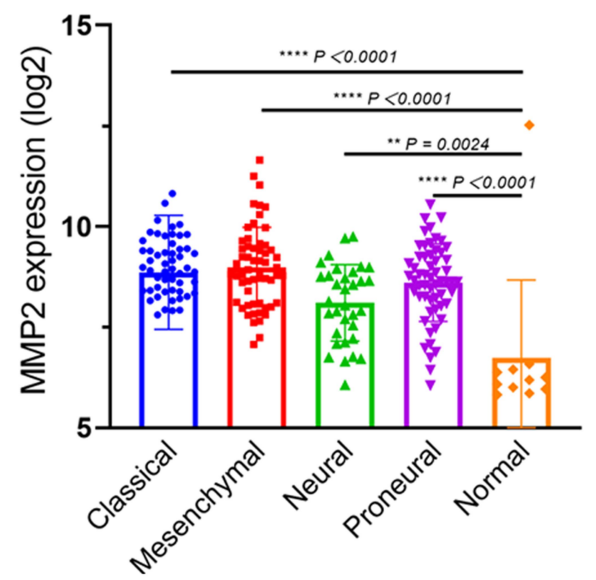

D

C

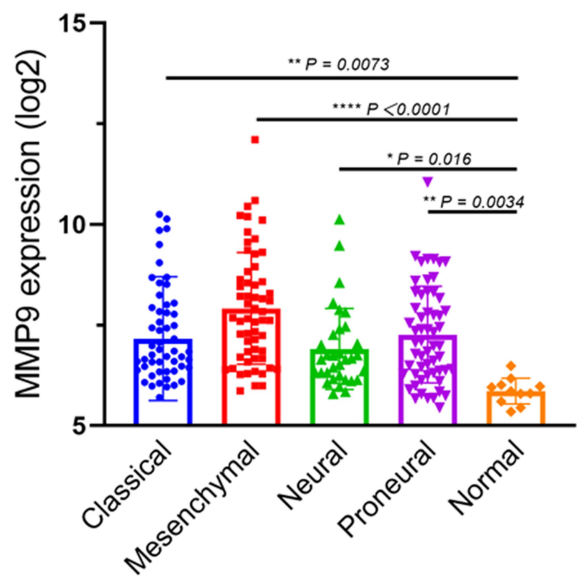

E

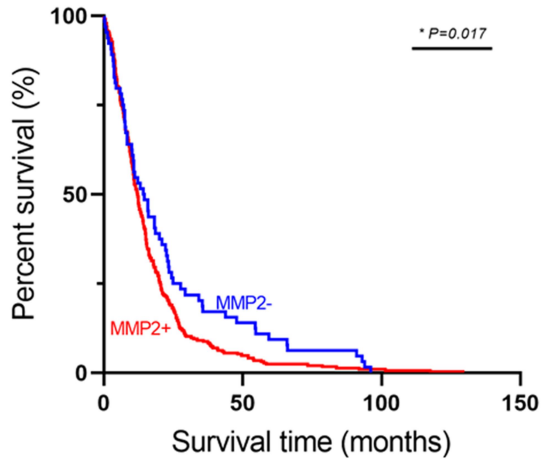

F

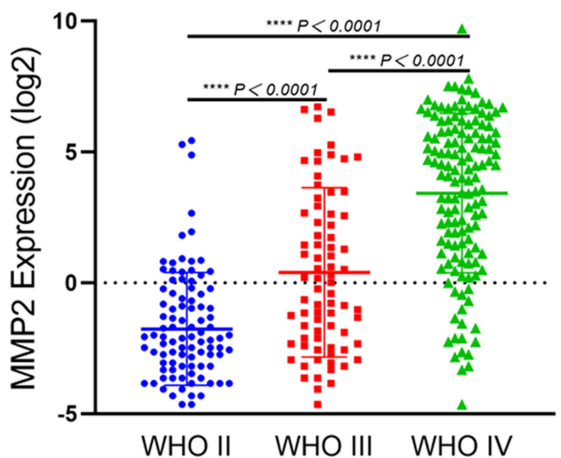

G
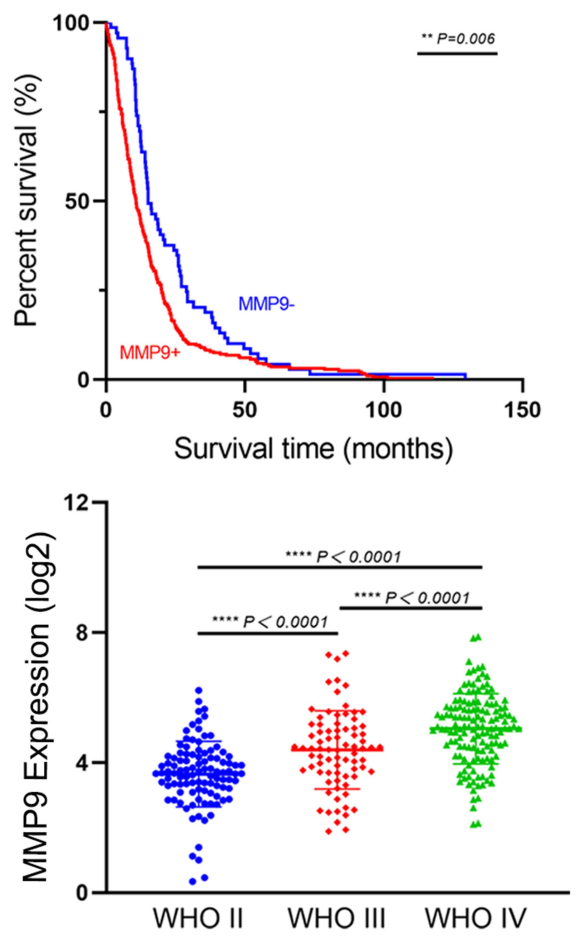

Figure I Association of MMP2 and MMP9 expression with patient survival. (A) Glioma tissue samples from patients were stained to visualize MMP2 and MMP9 (brown). Scale bar, $50 \mu \mathrm{m}$. (B and $\mathbf{C}$ ) The difference in MMP2 expression among different histological types of anaplastic gliomas patients and normal people, one-way ANOVA. (D and E) Kaplan-Meier survival curves for the glioma patients with low MMP2 expression (blue) compared to those with high MMP2 expression (red), Mantel-Cox Log rank test. ( $\mathbf{F}$ and $\mathbf{G}$ ) Expression of MMP2 protein level in different grade glioma, one-way ANOVA. 

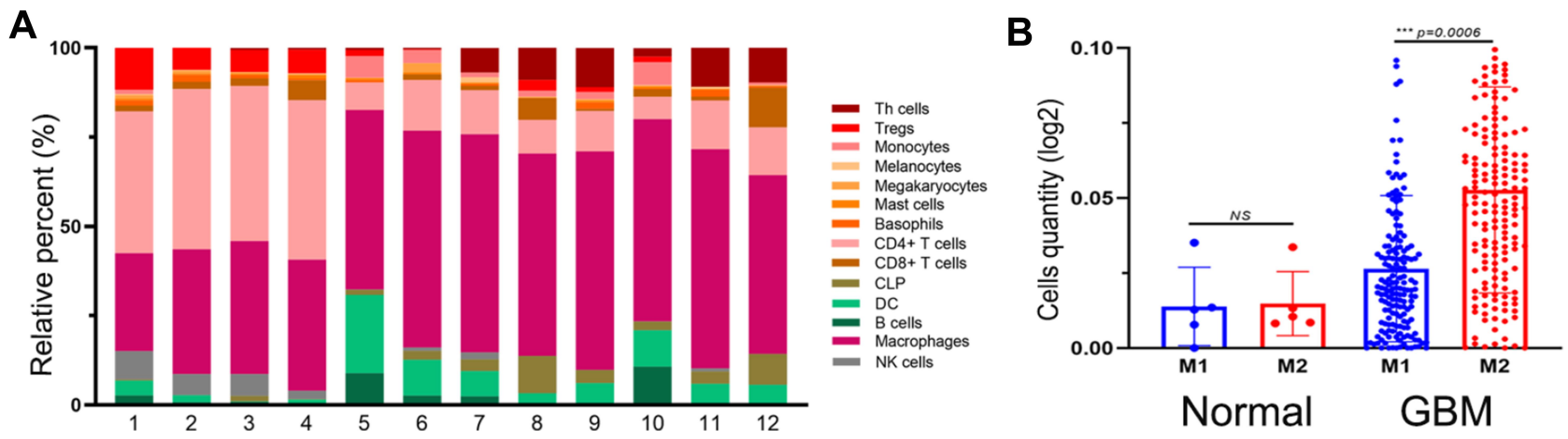

Figure $2 \mathrm{MI}$ and M2 macrophages expression in GBM. (A) Proportion of immune cells in normal and tumor tissues (I-4, normal; 5-12, GBM). (B) Expression of MI and M2 level in normal and GBM, $t$-test.

\section{Statistical Analysis}

All statistical analysis results were analyzed for differences between the two groups using the SPSS 19.0 software. When $\mathrm{P}<0.05$, it has statistical significance $\left({ }^{*} \mathrm{P}<0.05\right.$; $* * \mathrm{P}<0.01 ; * * * \mathrm{P}<0.001 ; * * * * \mathrm{P}<0.0001)$.

\section{Results}

\section{High MMPs Expression Correlates with Poor Glioma Patient Survival}

To clarify the clinicopathological significance of MMPs, we analyzed the expression of MMP2 and MMP9 in gliomas of different grades. We performed immunohistochemistry for MMP2 and MMP9 using samples from WHO grade II-III glioma and GBM. Compared with the normal group, the expression of MMP2 and MMP9 was obviously high in both grade II-III gliomas and GBM (Figure 1A). We then studied MMP2 and MMP9 expression in human glioma specimens using the CGGA database. When we explored MMP2 and MMP9 expression in different histological glioma subclasses, significantly more MMP2 and MMP9 in all types of glioma compared to the normal population (Figure 1B and C). Simultaneously, in the TCGA dataset, high MMP2 and MMP9 expression was associated with poor survival (Figure 1D and E). Furthermore, when we analyzed MMP2 and MMP9 expression in gliomas of different grades (grade II-IV), significantly more MMP2 and MMP9 were expressed in GBM samples compared to those from lower-grade gliomas (Figure $1 \mathrm{~F}$ and $\mathrm{G}$ ). All these results show that high MMP2 and MMP9 expression is associated with tumor progression and poor glioma patient survival.

\section{M2 Macrophages Constitute the Main Immune Cell Population in GBM}

TME is composed of multiple components including parenchyma cells, soluble factors, blood vessels, extracellular matrix, and infiltrating immune cells. ${ }^{18,34,35}$ M2 macrophages are often found in a huge proportion of malignant gliomas, which can suppress the immune response by reducing $\mathrm{CD} 4^{+}$and $\mathrm{CD} 8^{+} \mathrm{T}$ cell proliferation, enhancing tumor cell invasion and intravasation into blood vessels. ${ }^{36}$ Therefore, we assessed M1 and M2 macrophages in GBM using data obtained from the TCGA database. As expected, macrophages accounted for the highest proportion of all immune cells in tumor tissues, which was not obvious in normal tissues (Figure 2A). Moreover, M2 macrophages were more highly expressed in GBM than in M1 macrophages. In contrast, the difference in the expression of M1 and M2 in normal tissues was not statistically significant (Figure 2B).

\section{Preparation of MMPs Responsive G/Mino +Vor Hydrogels for Localized Delivery Drugs}

Initially, we added minocycline and vorinostat to the GelMA aqueous solution at $50^{\circ} \mathrm{C}$. The resultant solution, which also contained the photoinitiator, was then irradiated with $365 \mathrm{~nm}$ UV light to form a G/Mino+Vor hydrogel (Figure 3A). The surface of the G/Mino+Vor hydrogel was imaged by SEM (Figure 3B). GelMA has been reported to have a MMP-responsive property. ${ }^{37}$ Thus, we evaluated the ability of the G/Mino+Vor hydrogel to release the encapsulated minocycline and vorinostat in response to MMPs in vitro (Figure 3C). As 
A
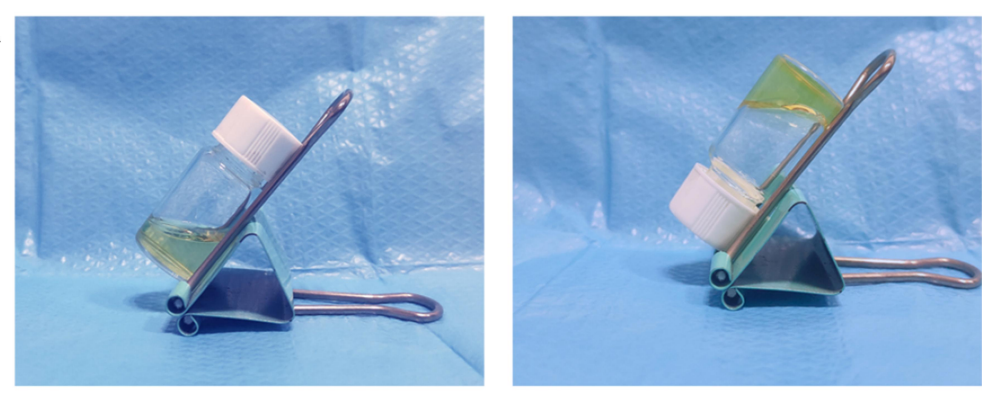

B

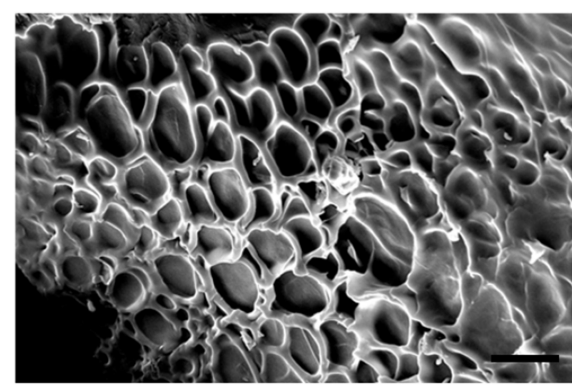

C
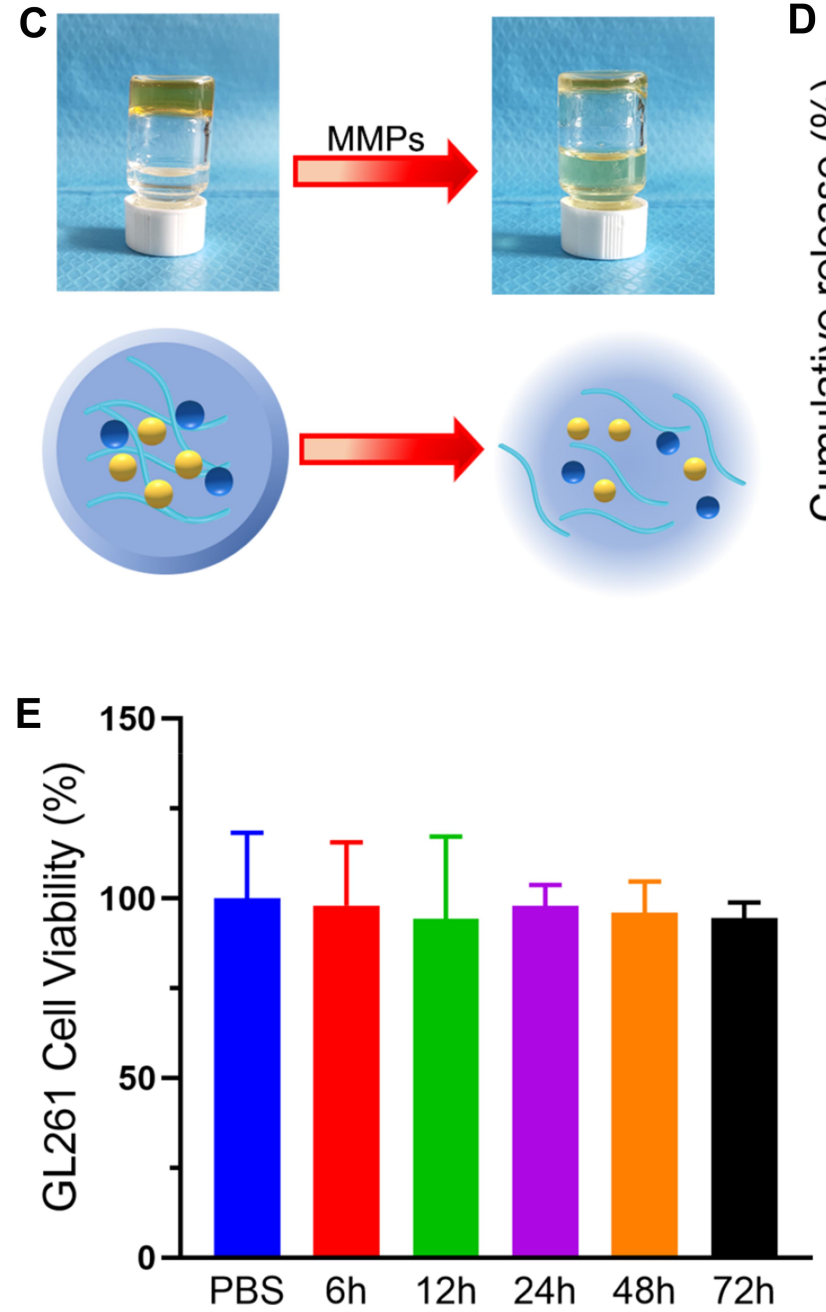

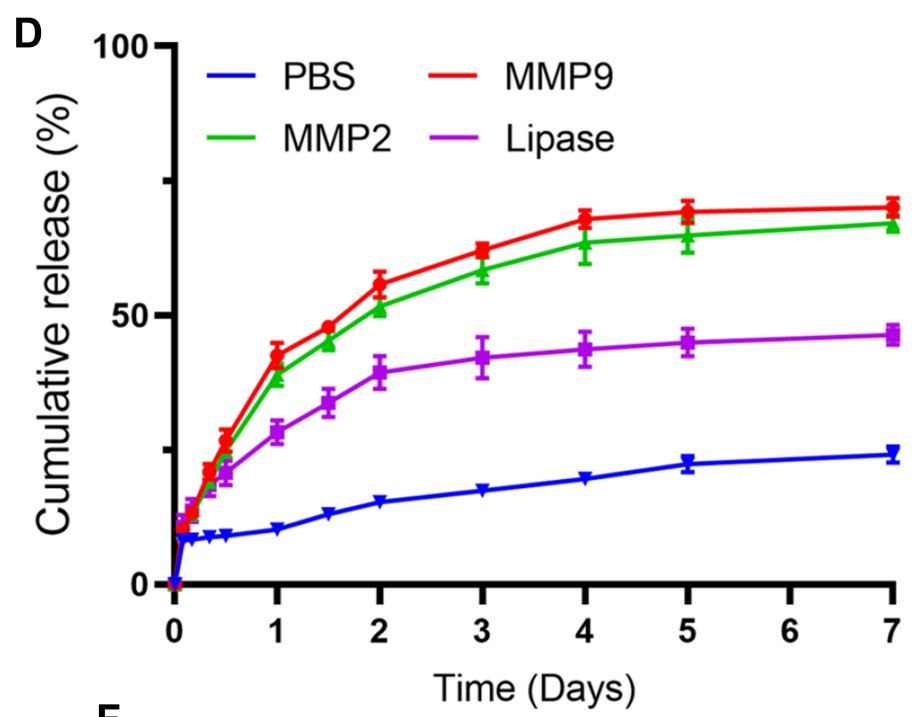

$\mathbf{F}$

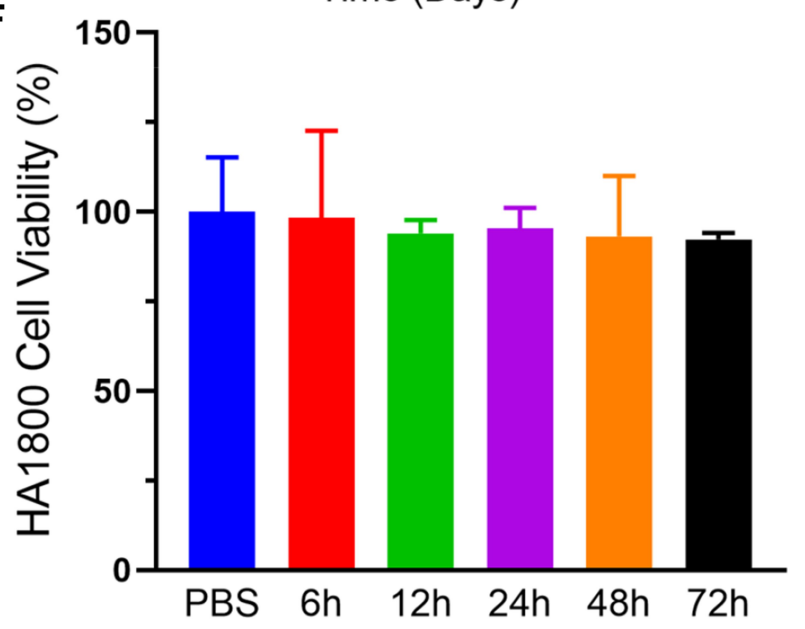

Figure 3 Preparation and characterization of G/Mino+Vor hydrogels. (A) Gelation of G/Mino+Vor hydrogel before and after UV irradiation. (B) SEM images of G/Mino+Vor hydrogel. Scale bar, $2 \mu \mathrm{m}$. (C) Schematic illustration of G/Mino+Vor hydrogel degradable process under MMPs conditions. (D) The drug release profile of G/Mino+Vor hydrogels under different conditions. (E and F) Toxicity detection of GelMA on GL26I and HAI800 cell lines.

expected, the addition of MMP2 and MMP9 caused an obviously increased release of drugs from the G/Mino + Vor hydrogel $(65.6 \%, 68.8 \%)$ compared with the PBS group (23.6\%); some effects were also observed with the lipase (Figure 3D). These results demonstrated that the
G/Mino+Vor hydrogel had good ability to release agents under MMPs conditions. Meanwhile, the cytotoxicity of GelMA was assessed. As shown in Figure 3E and Fand Fig S1, GelMA hydrogel was not toxic in either GL261 glioma cells or HA1800 cells. 
A

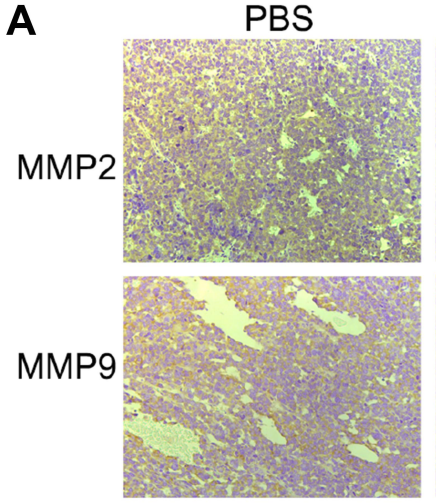

B
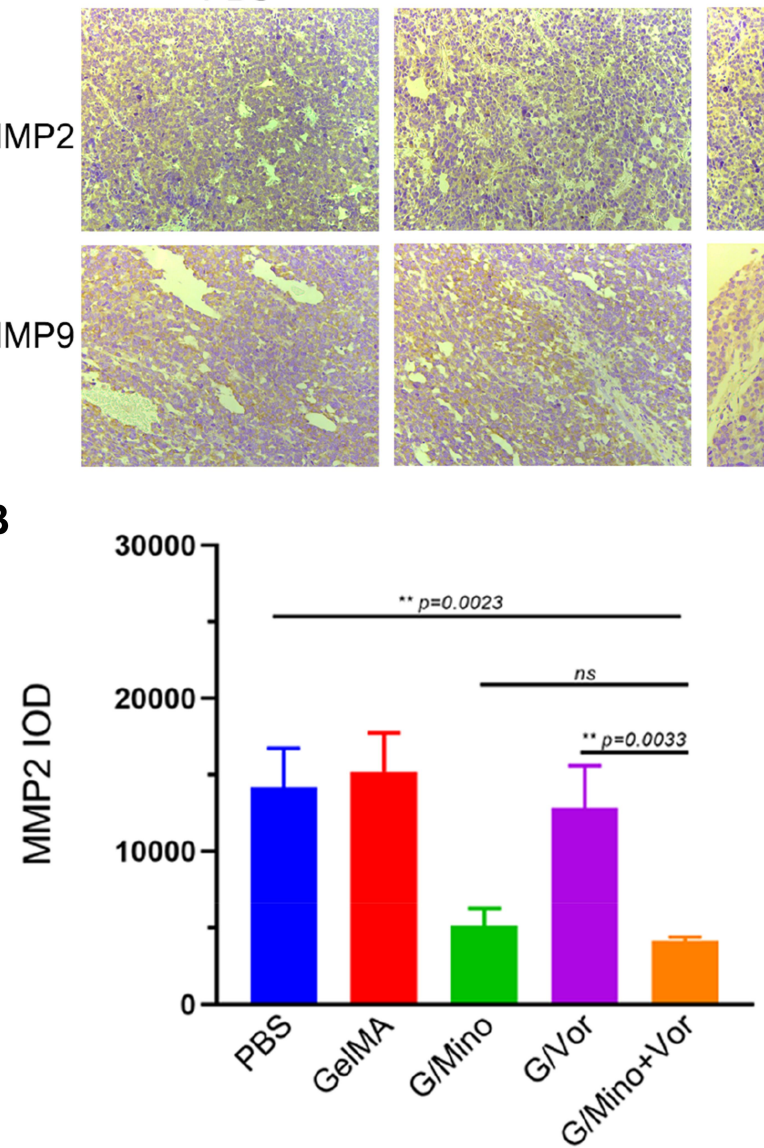

G/Mino

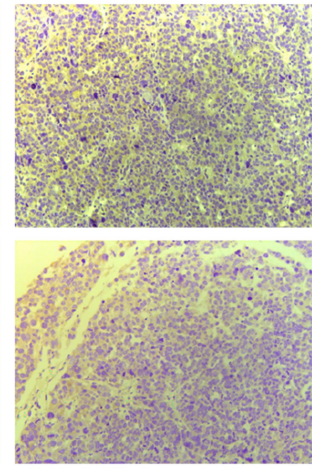

G/Nor

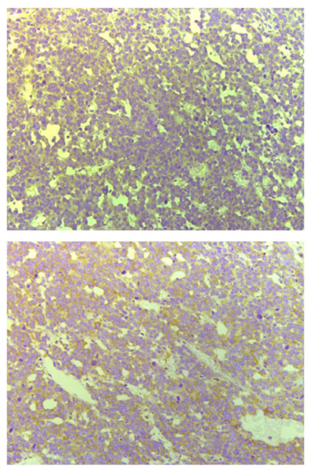

G/Mino+Vor

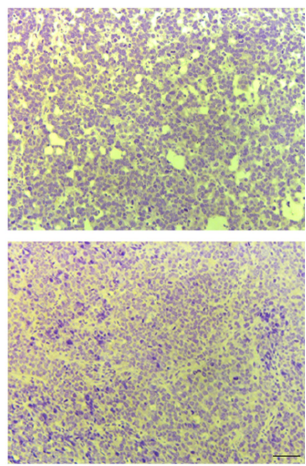

C

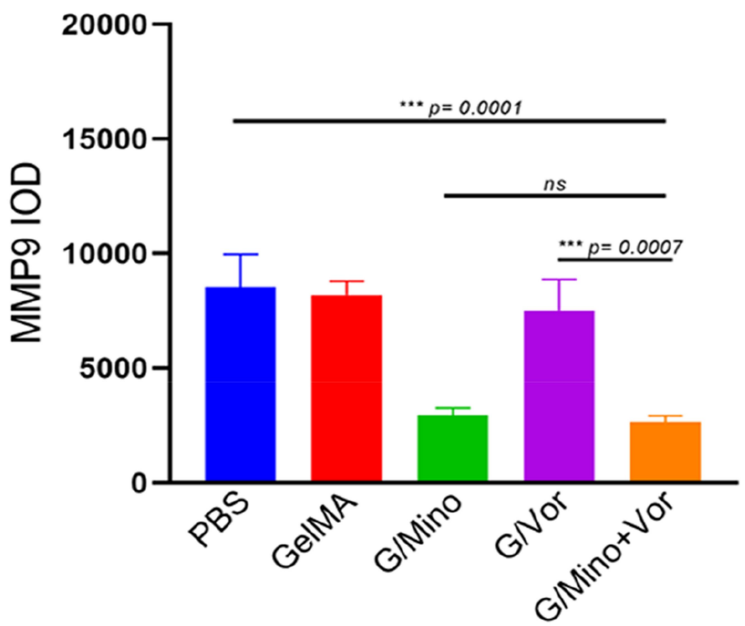

Figure 4 Reduced MMPs expression in response to G/Mino+Vor treatments. (A) Immunohistochemistry staining of representative tumor sections using MMP2 and MMP9 antibodies on the 28 thday after tumor implantation. Scale bar, $50 \mu \mathrm{m}$. (B and C) Immunohistochemical slides were analyzed quantitatively using integral optical density (IOD) analysis.

\section{G/Mino+Vor Treatment Reduced the Expression of MMPs}

We then evaluated the effect of in situ injection of the G/ Mino+Vor hydrogel on the lower expression of MMPs in mice bearing gliomas. We performed immunohistochemical staining using MMP2 and MMP9 antibody on the sections from the tumors (Figure 4A). MMP2 and MMP9 expression were assessed semi-quantitatively by integral optical density (IOD) analysis. Compared with the PBS group, the G/Mino and $\mathrm{G} / \mathrm{Mino}+$ Vor groups showed visible reduction in MMP2 and MMP9 expression, but there was no difference between the GelMA and G/Vor groups (Figure 4B and C).

\section{G/Mino+Vor Caused Hydrogel M2 Macrophage Reprogramming}

Next, to determine the effects of G/Mino+Vor on the repolarization of $\mathrm{M} 2$ to $\mathrm{M} 1$, we assessed markers for M1 and M2 macrophages using an ELISA kit. Compared with the PBS group, both G/Vor and G/Mino+Vor treatment drastically increased TNF- $\alpha$ expression in TAMs and improved their activity to produce IL-6, consistent with the M1 phenotype, which was not observed after treatment with GelMA and G/Mino (Figure 5A and B). Meanwhile, the expression levels of M2 macrophage markers IL-4 and IL-10 decreased, and no significant changes were observed in the GelMA and G/Mino groups (Figure 5C and D). Taken together, in addition to reducing the expression of MMP2 and MMP9, the G/Mino+Vor hydrogel could also lead to $\mathrm{M} 2$ repolarization.

\section{In vivo Anti-Glioma Recurrence of the G/ Mino+Vor Hydrogel}

To further explore the anti-tumor effect of minocycline and vorinostat released from the G/Mino+Vor hydrogel, we established an incomplete resection of the orthotopic glioma model. An incomplete operation was performed 

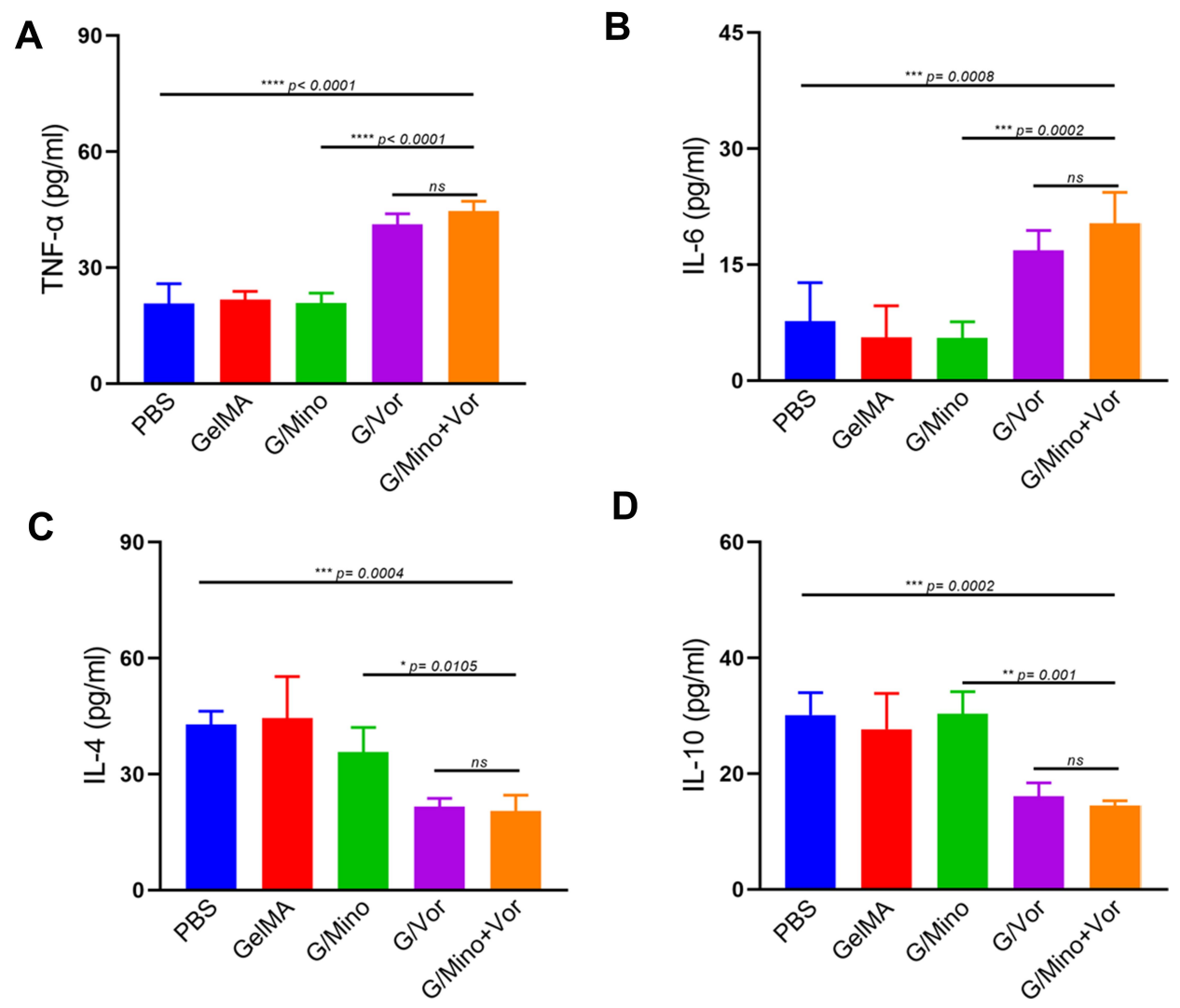

Figure 5 Repolarization of M2 macrophages caused by G/Mino+Vor treatments. (A-D) Levels of TNF- $\alpha$, IL-4, IL-6 and IL-10 in glioma tissue by ELISA on the 2 Ith day after tumor implantation. One-way ANOVA.

and the drug-loaded hydrogels were injected into the cavity to validate the therapeutic effects of the G/Mino +Vor hydrogel at day 10 post-tumor implantation. H\&E staining was used to stain the coronal section of the main organs of the mice at 28th day after implantation (Figure 6A). As expected, G/Mino+Vor hydrogel potently retarded tumor growth, while neither PBS nor GelMA treatment alone affected tumor growth, both G/Mino and G/Vor treatment partially slowed tumor growth (Figure 6B). Similarly, the G/Mino+Vor hydrogel-based therapy showed the best performance in extending the median survival time (38.5 days). The median survival times of groups PBS, GelMA, G/Mino, G/Vor were 22, 24, 27.5, and 28.5 days, respectively (Figure 6C). Changes in body weight were also evaluated. Not surprisingly, the body weights of mice in group G/Mino+Vor decreased slowly compared to the other treatments (Figure 6D). Simultaneously, the results of TUNEL staining indicated that apoptosis was significantly increased following G/Mino+Vor treatment compared with cells in other groups (Figure 6E).
Subsequently, the slices of major organs were stained with H\&E staining to evaluate toxicity in vivo. None of the groups exhibited any distinguishable abnormality (Figure 7). This demonstrated that the G/Mino+Vor hydrogel induced negligible systemic toxicity. Taken together, these findings show that the G/Mino+Vor hydrogel has potential value for clinical use.

\section{Discussion}

The formation of glioma produces a physiological microenvironment that inhibits the immune response and leads to the invasion of glioma cells. ${ }^{38}$ Thus TME, constituted with stromal cells, immune cells, and vasculatures, ${ }^{18}$ is becoming an emerging source of novel therapeutic targets cure in recent years. MMPs and macrophages have become popular topics among these targets.

MMPs are a alarge family of proteolytic enzymes that degrade ECM. ${ }^{11-13}$ The bioinformatics analysis results showed that the expression of MMPs is highly correlated with patient prognosis in our study. In addition, it has been reported that reducing the expression 

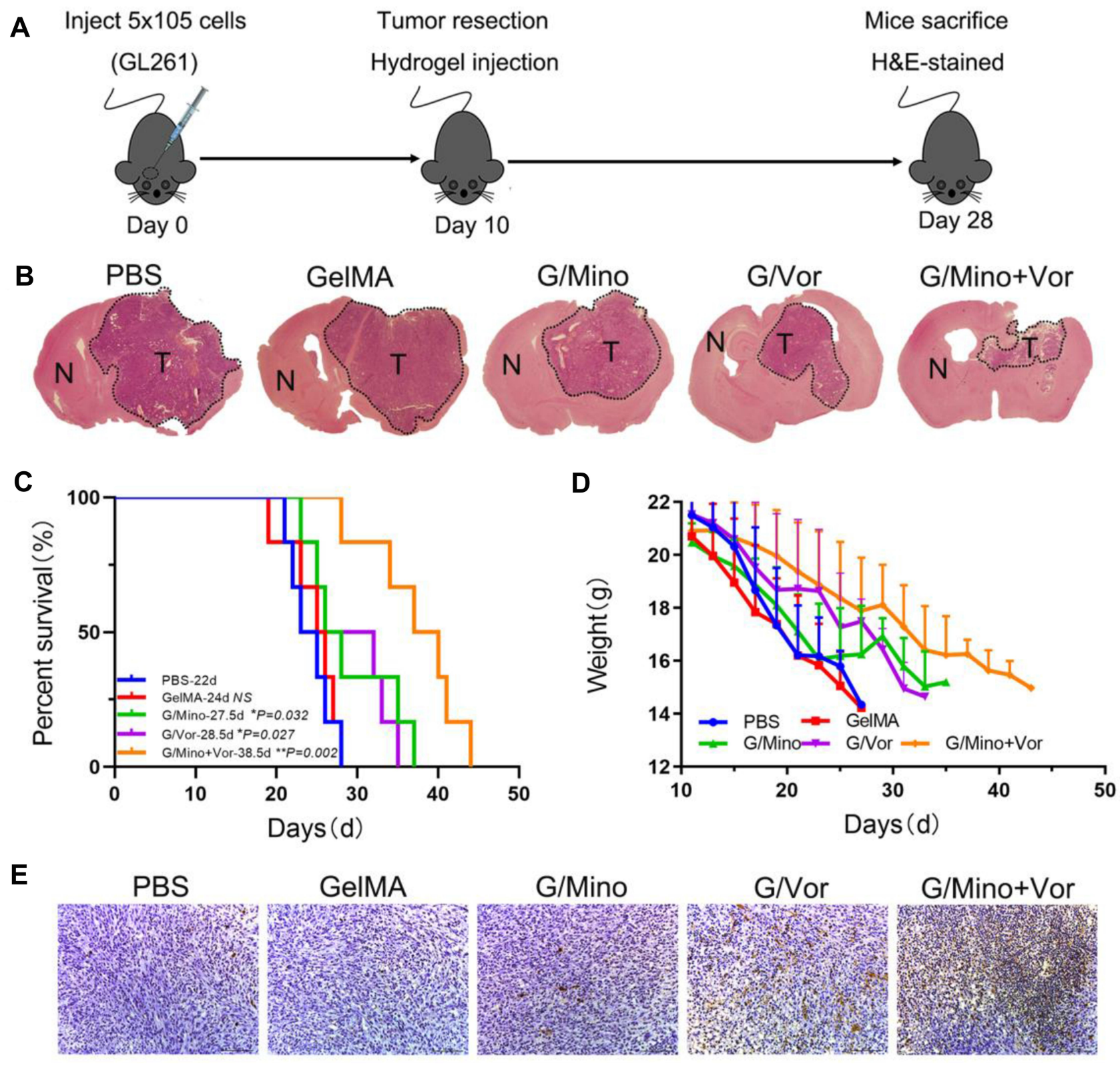

Figure 6 Therapeutic efficacy of G/Mino+Vor hydrogel in the GL26I glioma tumor model. (A) Schematic outline shows the in vivo experimental design for treatments. (B) Representative micrographs of H\&E stained tumors in orthotopic models. (C) Kaplan-Meier survival curves for the mice ( $n=6)$, one-way ANOVA. (D) Body weight change. Data are expressed as the mean \pm SEM $(n=6)$. (E) TUNEL staining of coronal sections from mouse brains with orthotopic tumors. Scale bar, $50 \mu m$.

of MMPs diminishes glioma proliferation, tumor growth, and neovascularization both in vitro and in vivo. ${ }^{39,40}$ In this study, we used minocycline to reduce its expression.

Macrophages play key roles in the glioma microenvironment. ${ }^{25}$ Increasing numbers of studies have shown that macrophages have different polarization states in different environments. In short, M1 macrophages promote the secretion of proinflammatory factors, whereas M2 macrophages can suppress the inflammatory reaction. ${ }^{41}$ Following the progression of glioma, M2 macrophages dominate the lesion microenvironment. Many researchers have pinpointed the polarization state of macrophages in the glioma microenvironment and used different approaches to influence it. Chen etal promoted the polarization of macrophages towards the M1 type by regulating the production of $\mathrm{ROS},{ }^{42}$ whereas Ma etal used dual-responsive polypeptide nano-vectors to deliver microRNA to achieve the same effect for anti-cancer therapy. ${ }^{43}$ 


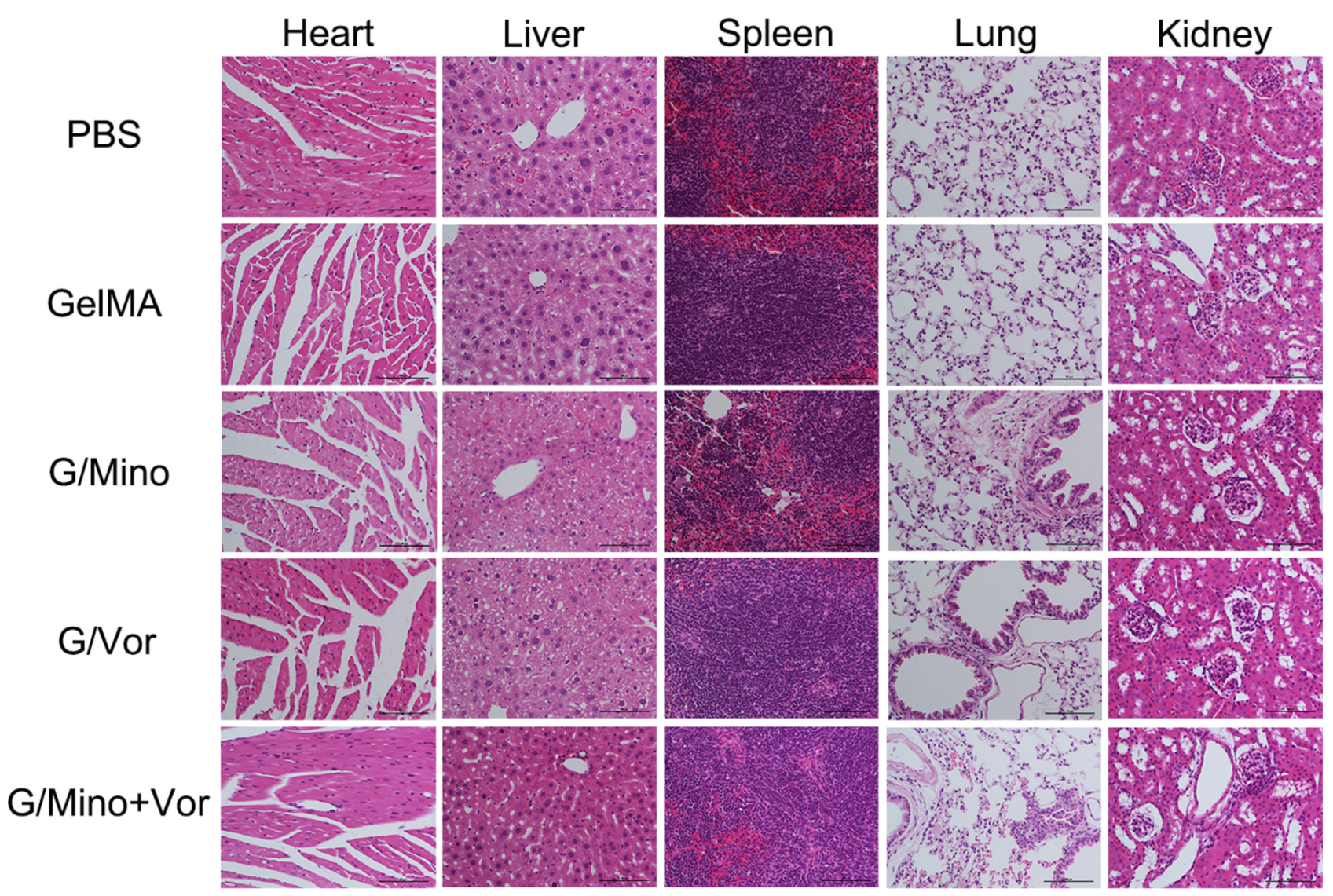

Figure 7 Toxicity analysis of G/Mino+Vor treatments. H\&E staining of heart, liver, spleen, lung and kidney from mice treated from different groups. Scale bar, $200 \mu$ m.

In contrast, we used vorinostat, which is a widely used HDACi. Early studies have already discovered that HDACi cause myelosuppression, thus implicating HDACi in monocyte/macrophage development. ${ }^{44}$ Given the broad effects of HDACi in regulating diverse cellular processes, HDACi can also amplify specific macrophage inflammatory responses. Trieu and Cao etal reported that HDACi enhanced LPS-inducible expression of Cox-225 and Pai-149 in macrophages to up-regulate the relative abundance of M1 macrophages. ${ }^{45,46}$ In addition, there are reports claiming that HDACi can also promote the repolarization of macrophages by regulating their metabolic status, ${ }^{47}$ but there is still much to learn about the signaling processes.

In this study, we developed an injectable G/Mino +Vor hydrogel that could induce the adjustment of the tumor microenvironment. G/Mino+Vor hydrogel represents a strategy, which can be injected at the time of incomplete surgical resection to deliver agents directly to the remnant tumor tissues. Thus, our injectable hydrogel has three advantages: (1) it minimized the system toxicity and improved biological safety; (2) it was able to maintain a steady release of the drugs in vivo over a long period of time; (3) it significantly increased the concentration of agents in residual tumor cells and enhanced the anti-glioblastoma activities.

In fact, the expression of MMPs was also reported to be associated with the amount of M2 macrophages, but the exact underlying molecular mechanism remains to be further investigated. Therefore, to clarify the mechanism of this phenomenon would be our future research endeavor.

\section{Conclusion}

In this study, an injectable biodegradable G/Mino+Vor hydrogel was successfully developed, which can be used to treat the recurrence of postoperative glioma. An in vitro release study showed that the release of MMPs gradually degraded the hydrogel. Then, drugs loaded in it, minocycline, and vorinostat could reshape TME. In vivo experiments showed that the G/Mino+Vor hydrogel inhibits recurrence of glioma cancer progression. Additionally, 
A

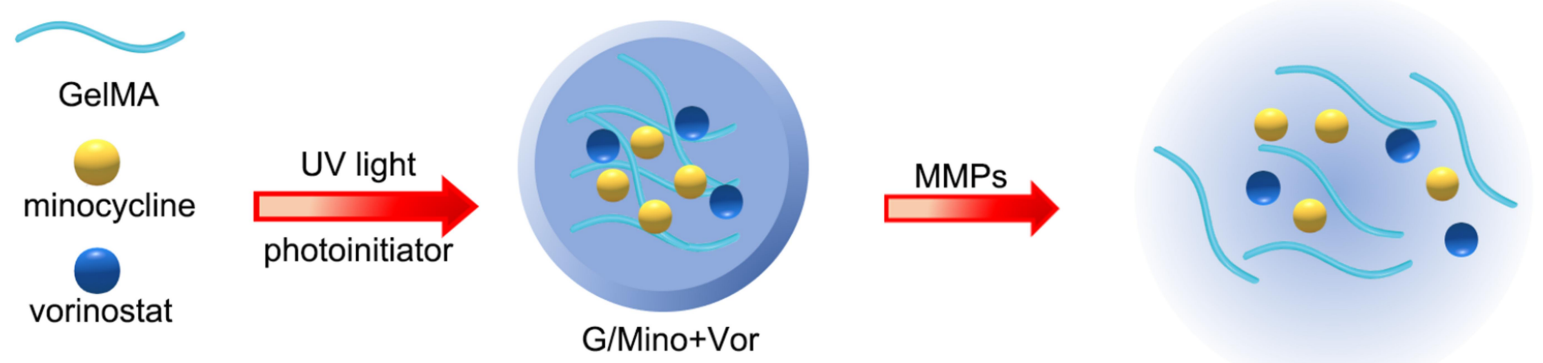

B

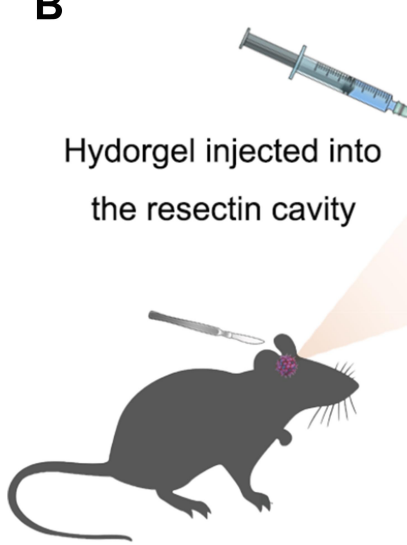

Surgical resection

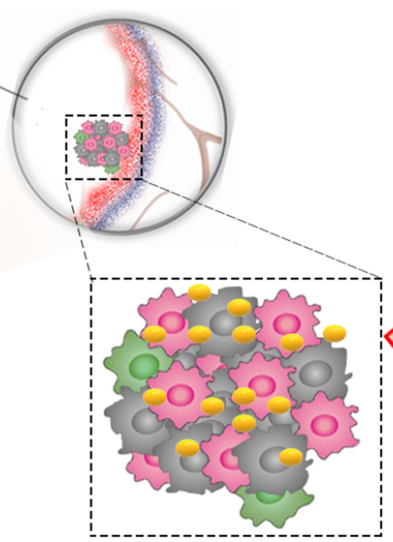

M1 macrophages

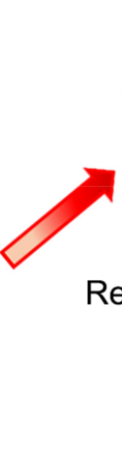

Repolarizing M2 to M1

Reducing expression of MMPs

M2 macrophages MMPs

Scheme I The structure of G/Mino+Vor hydrogel and its effects in inhibiting the recurrence of glioma. (A) The G/Mino+Vor hydrogel is formed under UV light and corresponds to MMPs. (B) Minocycline and vorinostat released from G/Mino+Vor hydrogel under post-operative environment, which could repolarize M2 to MI and reduce expression of MMPs.

the secretion of MMP2 and MMP9 decreased and M2 to M1 repolarization was observed. In summary, this suggests that the $\mathrm{G} / \mathrm{Mino}+$ Vor hydrogel may be utilized as a potential formulation strategy to restrain the recurrence of residual glioma after surgery.

\section{Abbreviation}

Gelatin methacryloyl (GelMA or G); minocycline (Mino); vorinostat (Vor); central nervous system (CNS); glioblastoma (GBM); extracellular matrix (ECM); matrix metalloproteinases (MMPs); toll like receptor 2 (TLR2); mitogenactivated protein kinase (MAPK); blood-brain barrier (BBB); tumor microenvironment (TME); Phosphate Buffered Saline (PBS); Fetal Bovine Serum (FBS); Dulbecco's Modified Eagle Medium (DMEM); optical density (OD); bovine serum (BSA); Diaminobenzidine (DAB); tumor necrosis factor (TNF); interleukin (IL); enzyme-linked immunosorbent assay (ELISA).

\section{Acknowledgments}

We thank Rutong Yu in Department of Neurosurgery, Affiliated Hospital of Xuzhou Medical University for his critical assistance to this work, as great as the existing correspondence authors, Hongmei Liu and Yufu Zhu.

\section{Funding}

This work was financially supported by National Natural Science Foundation of China (No. 81772665), Natural Science Foundation of Jiangsu Province (No. BK20181149). This paper was also financed from Jiangsu Provincial Commission of Health and Family Planning (No. Q201608). Research was supported by Six Talents Peak Foundation of Jiangsu Province (No. 2018-WSW-071). This work was supported by a grant from Postgraduate Research \& Practice Innovation Program of Jiangsu Province (No. KYCX192236), Social Development Project of Jiangsu Department of Science and Technology (No. BE2020647). 


\section{Disclosure}

The authors declare that they have no competing interests.

\section{References}

1. Meyer MA. Malignant gliomas in adults. $N$ Engl J Med. 2008;359 (17): 1850 ; author reply 1850 .

2. Stupp R, Mason WP, van den Bent MJ, et al. European organisation for, R.; treatment of cancer brain, T.; radiotherapy, G.; national cancer institute of canada clinical trials, G., radiotherapy plus concomitant and adjuvant temozolomide for glioblastoma. $N$ Engl $J$ Med. 2005;352(10):987-996. doi:10.1056/NEJMoa043330

3. Nicholas S, Mathios D, Ruzevick J, Jackson C, Yang I, Lim M. Current trends in glioblastoma multiforme treatment: radiation therapy and immune checkpoint inhibitors. Brain Tumor Res Treat. 2013;1(1):2-8. doi:10.14791/btrt.2013.1.1.2

4. Templeton A, Hofer S, Topfer M, et al. Extraneural spread of glioblastoma-report of two cases. Onkologie. 2008;31(4):192-194. doi:10.1159/000118627

5. Ahmed R, Oborski M, Hwang M, Lieberman F, Mountz J. Malignant gliomas: current perspectives in diagnosis, treatment, and early response assessment using advanced quantitative imaging methods. Cancer Management and Research. 2014;6:149-170.

6. Gilbert MR, Dignam JJ, Armstrong TS, et al. A randomized trial of bevacizumab for newly diagnosed glioblastoma. $N$ Engl $J$ Med. 2014;370(8):699-708. doi:10.1056/NEJMoa1308573

7. Gilbert MR, Wang M, Aldape KD, et al. Dose-dense temozolomide for newly diagnosed glioblastoma: a randomized Phase III clinical trial. J Clin Oncol. 2013;31(32):4085-4091. doi:10.1200/JCO.2013.49.6968

8. Palmer JD, Bhamidipati D, Song A, et al. Bevacizumab and re-irradiation for recurrent high grade gliomas: does sequence matter? J Neurooncol. 2018;140(3):623-628. doi:10.1007/s11060-018-2989-z

9. Sharma RR, Singh DP, Pathak A, et al. Local control of high-grade gliomas with limited volume irradiation versus whole brain irradiation. Neurol India. 2003;51(4):512-517.

10. Kadiyala P, Li D, Nunez FM, et al. High-density lipoprotein-mimicking nanodiscs for chemo-immunotherapy against glioblastoma multiforme. ACS Nano. 2019;13(2):1365-1384.

11. Osenkowski P, Toth M, Fridman R. Processing, shedding, and endocytosis of membrane type 1-matrix metalloproteinase (MT1-MMP). $J$ Cell Physiol. 2004;200(1):2-10. doi:10.1002/jcp.20064

12. Markovic DS, Vinnakota K, van Rooijen N, et al. Minocycline reduces glioma expansion and invasion by attenuating microglial MT1-MMP expression. Brain Behav Immun. 2011;25(4):624-628. doi:10.1016/j.bbi.2011.01.015

13. Rao JS. Molecular mechanisms of glioma invasiveness: the role of proteases. Nat Rev Cancer. 2003;3(7):489-501. doi:10.1038/nrc1121

14. Du R, Lu KV, Petritsch C, et al. HIF1alpha induces the recruitment of bone marrow-derived vascular modulatory cells to regulate tumor angiogenesis and invasion. Cancer Cell. 2008;13(3):206-220. doi:10.1016/j.ccr.2008.01.034

15. Bergers G, Brekken R, McMahon G, et al. Matrix metalloproteinase-9 triggers the angiogenic switch during carcinogenesis. Nat Cell Biol. 2000;2(10):737-744.

16. Smith SJ, Tyler BM, Gould T, et al. Overall survival in malignant glioma is significantly prolonged by neurosurgical delivery of etoposide and temozolomide from a thermo-responsive biodegradable paste. Clinical Cancer Res. 2019;25(16):5094. doi:10.1158/10780432.CCR-18-3850

17. Urban TJ, Nicoletti P, Chalasani N, et al. Minocycline hepatotoxicity: clinical characterization and identification of HLA-B*35:02 as a risk factor. J Hepatol. 2017;67(1):137-144. doi:10.1016/j.jhep.2017.03.010

18. Chanmee T, Ontong P, Konno K, Itano N. Tumor-associated macrophages as major players in the tumor microenvironment. Cancers (Basel). 1670-1690;2014(6):3.
19. Burkholder B, Huang RY, Burgess R, et al. Tumor-induced perturbations of cytokines and immune cell networks. Biochim Biophys Acta. 2014;1845(2):182-201.

20. Mosser DM, Edwards JP. Exploring the full spectrum of macrophage activation. Nat Rev Immunol. 2008;8(12):958-969. doi:10.1038/ nri2448

21. Riabov V, Gudima A, Wang N, Mickley A, Orekhov A, Kzhyshkowska J. Role of tumor associated macrophages in tumor angiogenesis and lymphangiogenesis. Front Physiol. 2014;5:75. doi:10.3389/fphys.2014.00075

22. Mills CD, Lenz LL, Harris RA, Breakthrough A. MacrophageDirected Cancer Immunotherapy. Cancer Research. 2016;76 (3):513-516. doi:10.1158/0008-5472.CAN-15-1737

23. Hanahan D, Coussens LM. Accessories to the crime: functions of cells recruited to the tumor microenvironment. Cancer Cell. 2012;21 (3):309-322. doi:10.1016/j.ccr.2012.02.022

24. Guerriero JL, Sotayo A, Ponichtera HE, et al. Class IIa HDAC inhibition reduces breast tumours and metastases through anti-tumour macrophages. Nature. 2017;543(7645):428-432. doi:10.1038/nature21409

25. Peng H, Chen B, Huang W, et al. Reprogramming tumor-associated macrophages to reverse EGFR (T790M) resistance by dual-targeting codelivery of gefitinib/vorinostat. Nano Lett. 2017;17 (12):7684-7690. doi:10.1021/acs.nanolett.7b03756

26. Tran K, Risingsong R, Royce DB, et al. The combination of the histone deacetylase inhibitor vorinostat and synthetic triterpenoids reduces tumorigenesis in mouse models of cancer. Carcinogenesis. 2013;34(1):199-210. doi:10.1093/carcin/bgs319

27. Ramalingam SS, Maitland ML, Frankel $P$, et al. Carboplatin and Paclitaxel in combination with either vorinostat or placebo for first-line therapy of advanced non-small-cell lung cancer. $J$ Clin Oncol. 2010;28(1):56-62. doi:10.1200/JCO.2009.24.9094

28. Kelly WK, O'Connor OA, Krug LM, et al. Phase I study of an oral histone deacetylase inhibitor, suberoylanilide hydroxamic acid, in patients with advanced cancer. $J$ Clin Oncol. 2005;23 (17):3923-3931. doi:10.1200/JCO.2005.14.167

29. Choi BY, Chalisserry EP, Kim MH, Kang HW, Choi IW, Nam SY. The influence of astaxanthin on the proliferation of adipose-derived mesenchymal stem cells in gelatin-methacryloyl (gelma) hydrogels. Materials (Basel. 2019;12:15. doi:10.3390/ma12152416

30. Nichol JW, Koshy ST, Bae H, Hwang CM, Yamanlar S, Khademhosseini A. Cell-laden microengineered gelatin methacrylate hydrogels. Biomaterials. 2010;31(21):5536-5544. doi:10.1016/j. biomaterials.2010.03.064

31. Billiet T, Gevaert E, De Schryver T, Cornelissen M, Dubruel P. The 3D printing of gelatin methacrylamide cell-laden tissue-engineered constructs with high cell viability. Biomaterials. 2014;35(1):49-62. doi:10.1016/j.biomaterials.2013.09.078

32. Yue K, Trujillo-de Santiago G, Alvarez MM, Tamayol A, Annabi N, Khademhosseini A. Synthesis, properties, and biomedical applications of gelatin methacryloyl (GelMA) hydrogels. Biomaterials. 2015;73:254-271. doi:10.1016/j.biomaterials.2015.08.045

33. Liu W, Zhong Z, Hu N, et al. Coaxial extrusion bioprinting of 3D microfibrous constructs with cell-favorable gelatin methacryloyl microenvironments. Biofabrication. 2018;10(2):024102. doi:10.1088/1758-5090/aa9d44

34. Kim J, Bae JS. Tumor-associated macrophages and neutrophils in tumor microenvironment. Mediators Inflamm. 2016;2016:6058147. doi: $10.1155 / 2016 / 6058147$

35. Van Overmeire E, Laoui D, Keirsse J, Van Ginderachter JA, Sarukhan A. Mechanisms driving macrophage diversity and specialization in distinct tumor microenvironments and parallelisms with other tissues. Front Immunol. 2014;5:127.

36. Kelly PM, Davison RS, Bliss E, McGee JO. Macrophages in human breast disease: a quantitative immunohistochemical study. $\mathrm{Br}$ J Cancer. 1988;57(2):174-177. doi:10.1038/bjc.1988.36 
37. Luo Z, Sun W, Fang J, et al. Biodegradable gelatin methacryloyl microneedles for transdermal drug delivery. Adv Healthc Mater. 2019;8(3):e1801054. doi:10.1002/adhm.201801054

38. Fang T, Lv H, Lv G, et al. Tumor-derived exosomal miR-1247-3p induces cancer-associated fibroblast activation to foster lung metastasis of liver cancer. Nature communications. 2018;9(1):191-191.

39. Zhao Y, Xiao A, diPierro CG, et al. An extensive invasive intracranial human glioblastoma xenograft model: role of high level matrix metalloproteinase 9. Am J Pathol. 2010;176(6):3032-3049.

40. Sun C, Wang Q, Zhou H, et al. Antisense MMP-9 RNA inhibits malignant glioma cell growth in vitro and in vivo. Neurosci Bull. 2013;29(1): 83-93.

41. Kroner A, Greenhalgh AD, Zarruk JG, Passos Dos Santos R, Gaestel M, David S. TNF and increased intracellular iron alter macrophage polarization to a detrimental M1 phenotype in the injured spinal cord. Neuron. 2014;83(5):1098-1116.

42. Shi C, Liu T, Guo Z, Zhuang R, Zhang X, Chen X. Reprogramming Tumor-Associated Macrophages by Nanoparticle-Based Reactive Oxygen Species Photogeneration. Nano Lett. 2018;18(11):7330 7342
43. Liu L, Yi H, He H, Pan H, Cai L, Ma Y. Tumor associated macrophage-targeted microRNA delivery with dual-responsive polypeptide nanovectors for anti-cancer therapy. Biomaterials. 2017;134:166179.

44. Bishton MJ, Harrison SJ, Martin BP, et al. Deciphering the molecular and biologic processes that mediate histone deacetylase inhibitorinduced thrombocytopenia. Blood. 2011;117(13):3658-3668.

45. Aung HT, Schroder K, Himes SR, et al. LPS regulates proinflammatory gene expression in macrophages by altering histone deacetylase expression. FASEB journal : official publication of the Federation of American Societies for Experimental Biology. 2006;20(9):13151327.

46. Halili MA, Andrews MR, Labzin LI, et al. Differential effects of selective HDAC inhibitors on macrophage inflammatory responses to the Toll-like receptor 4 agonist LPS. Journal of leukocyte biology. 2010;87(6):1103-1114.

47. Van den Bossche J, Neele AE, Hoeksema MA, et al. Inhibiting epigenetic enzymes to improve atherogenic macrophage functions. Biochem Biophys Res Commun. 2014;455(3-4):396-402.

\section{Publish your work in this journal}

OncoTargets and Therapy is an international, peer-reviewed, open access journal focusing on the pathological basis of all cancers, potential targets for therapy and treatment protocols employed to improve the management of cancer patients. The journal also focuses on the impact of management programs and new therapeutic agents and protocols on patient perspectives such as quality of life, adherence and satisfaction. The manuscript management system is completely online and includes a very quick and fair peer-review system, which is all easy to use. Visit http://www.dovepress.com/ testimonials.php to read real quotes from published authors. 